PROCEEDINGS OF THE

AMERICAN MATHEMATICAL SOCIETY

Volume 127, Number 11, Pages 3141-3151

S 0002-9939(99)05199-0

Article electronically published on May 4, 1999

\title{
FINITE GENERATION OF POWERS OF IDEALS
}

\author{
ROBERT GILMER, WILLIAM HEINZER, AND MOSHE ROITMAN
}

(Communicated by Wolmer V. Vasconcelos)

\begin{abstract}
Suppose $M$ is a maximal ideal of a commutative integral domain $R$ and that some power $M^{n}$ of $M$ is finitely generated. We show that $M$ is finitely generated in each of the following cases: (i) $M$ is of height one, (ii) $R$ is integrally closed and ht $M=2$, (iii) $R=K[X ; \tilde{S}]$ is a monoid domain over a field $K$, where $\tilde{S}=S \cup\{0\}$ is a cancellative torsion-free monoid such that $\bigcap_{m=1}^{\infty} m S=\emptyset$, and $M$ is the maximal ideal $\left(X^{s}: s \in S\right)$. We extend the above results to ideals $I$ of a reduced ring $R$ such that $R / I$ is Noetherian. We prove that a reduced ring $R$ is Noetherian if each prime ideal of $R$ has a power that is finitely generated. For each $d$ with $3 \leq d \leq \infty$, we establish existence of a $d$-dimensional integral domain having a nonfinitely generated maximal ideal $M$ of height $d$ such that $M^{2}$ is 3 -generated.
\end{abstract}

\section{INTRODUCTION}

All rings in this paper are commutative with unity. We consider the following question:

Question 0.1. Suppose that some power $M^{n}$ of the maximal ideal $M$ of an integral domain $R$ is finitely generated. Does it follow that $M$ is finitely generated?

This question is raised in [3, page 74] and was mentioned in a talk given by the first author at the AMS meeting in Auburn, Alabama in November 1971. It is also listed, for the case of a quasilocal integrally closed domain, as Problem 8 in the questions list on pages 174-176 in the 1973 Notices of the AMS from the problem session organized by Graham Evans at the January 1973 AMS meeting in Dallas.

We answer Question 0.1 in the negative in $\S 3$. We also consider the following generalization of Question 0.1.

Question 0.2. Suppose $I$ is an ideal of a ring $R$ such that $R / I$ is Noetherian. If some power of $I$ is finitely generated, under what conditions is $I$ finitely generated?

In $\S 1$ we present positive results on these two questions. Regarding Question 0.1 we prove in Theorem 1.24, among other results, that the maximal ideal $M$ is finitely generated under each of the following conditions: (i) $R$ is a reduced ring

Received by the editors January 26, 1998.

1991 Mathematics Subject Classification. Primary 13A15, 13E99, 13G05.

Key words and phrases. Cohen's theorem, finite generation, maximal ideal, monoid ring, Noetherian, power of an ideal, Ratliff-Rush closure.

The first two authors acknowledge with thanks the hospitality of the mathematics department of the University of North Carolina at Chapel Hill. Partial support of the work of the second author by the National Science Foundation is also gratefully acknowledged.

(C)1999 American Mathematical Society 
and ht $M=1$, (ii) $R$ is an integrally closed domain and ht $M=2$. We would like to know the answer to Question 0.1 if $R$ is an integrally closed (or even completely integrally closed) domain, eventually assuming that $M$ is of finite height.

Regarding Question 0.2 we prove in Theorem 1.10, among other results, that $I$ is finitely generated if $I$ is the radical of an ideal generated by a nonempty regular sequence. Also in $\S 1$ we present for the case of a reduced ring an analogue to Cohen's Theorem [8, (3.4)]. We assume in Theorem 1.17 that each prime ideal of a reduced ring $R$ has a power that is finitely generated and conclude that $R$ is Noetherian. Moreover, if $R$ is semiquasilocal, we prove that $R$ is Noetherian if each prime of $R$ of positive height has a finitely generated power (Theorem 1.20).

The context in which Question 0.1 was raised in [3] makes it natural to consider the question in a monoid domain over a field. Thus in $\S 2$ we prove that the analogue of Question 0.1 for a commutative cancellative semigroup $S$ has a positive answer provided $\bigcap_{m=1}^{\infty} m S=\emptyset$ (Theorem 2.3). We establish in Proposition 2.1 the connection between these two aspects of Question 0.1 (for semigroups and for rings), and this allows us to conclude in Theorem 2.5 that if $R=K[X ; T]$ is a monoid ring over a field $K$, where $T$ is a cancellative monoid, $S=T \backslash\{0\}, \bigcap_{m=1}^{\infty} m S=\emptyset$, and $M$ is the maximal ideal $\left(X^{s}: s \in S\right)$, then $M$ is finitely generated if some power of $M$ is finitely generated. Without the restriction $\bigcap_{m=1}^{\infty} m S=\emptyset$, we show in Example 3.2 that the ideal $M$ need not be finitely generated.

\section{Powers of ideAls}

The main tool for dealing with Questions 0.1 and 0.2 is Theorem 1.9, which states that under the hypothesis of Question 0.2 the ideal $I$ is finitely generated if $I$ is the radical of a principal ideal and $c I \neq(0)$ for each nonzero $c \in I$. The proof of Theorem 1.9 is based on Lemma 1.4. Moreover, Lemma 1.8 reduces Question 0.1 to the case of quasilocal rings, and Lemma 1.7 reduces Question 0.2 to the case of radical ideals.

Lemma 1.1. Let $J \subseteq I$ be ideals in a ring $R$ such that $I^{n}=J^{n}$ for some positive integer $n$. Then $I^{m}=I^{i} J^{m-i}$ for all $m \geq n$ and $0 \leq i \leq m$.

Proof. Since $J \subseteq I$, the ideal $I^{i} J^{m-i}$ lies between $I^{m}$ and $J^{m}$. Thus it suffices to show $I^{m}=J^{m}$. Moreover, $I^{n}=J I^{n-1}$ implies $I^{n+1}=J I^{n}=J^{n+1}$. The lemma follows by induction.

Lemma 1.2. If $I$ is an ideal of a ring $R$ such that $I^{n}$ is finitely generated for some $n \geq 1$, then there exists a finitely generated ideal $J \subseteq I$ such that $I^{n}=J^{n}$.

Proof. Let $I^{n}=\left(a_{1}, \ldots, a_{k}\right)$. By definition of $I^{n}$, the element $a_{j}$ is a finite sum of products of $n$ elements of $I$, and hence belongs to $B_{j}^{n}$ for some finitely generated subideal $B_{j}$ of $I$. Set $J=\sum B_{j}$.

Remark 1.3. Suppose $M$ is a maximal ideal of a ring $R$ such that $M^{n}$ is finitely generated for some $n \geq 1$. Lemmas 1.1 and 1.2 imply that there exists a finitely generated ideal $I \subseteq M$ such that $M$ is the Ratliff-Rush closure of $I$ in the sense that $M$ is the largest ideal containing $I$ and having the same high powers as $I$ [10], [6]. In particular, $M$ is integral over $I$.

In connection with generators of powers of an ideal, Eakin and Sathaye prove in $[1$, page 440] the interesting result that if $I$ is an ideal of a quasilocal ring with infinite residue field and if $n$ and $r$ are positive integers such that $I^{n}$ can be 
generated by fewer than $\left(\begin{array}{l}n \\ r\end{array}\right)$ elements, then there exists an $r$-generated ideal $J \subseteq I$ such that $J I^{n-1}=I^{n}$.

Lemma 1.4. Let $N \subseteq K \subseteq M$ be modules over a ring $R$ such that $N=\left(\prod_{j=1}^{k} I_{j}\right) M$, where $I_{j}$ is an ideal of $R$ so that $R / I_{j}$ is Noetherian for $1 \leq j \leq k$. Assume that all the R-modules $\left(\prod_{j=1}^{e} I_{j}\right) M$ for $0 \leq e \leq k$ are finitely generated (for $e=0$ we have $\prod_{j=1}^{e} I_{j}=R$ ). Then the $R$-module $K$ is finitely generated.

Proof. Consider the sequence of $R$-modules: $N=\left(\prod_{j=1}^{k} I_{j}\right) M \subseteq\left(\prod_{j=1}^{k-1} I_{j}\right) M \subseteq$ $\cdots \subseteq I_{1} M \subseteq M$. Each quotient of two successive modules in this sequence is a finitely generated module over the Noetherian ring $R / I_{j}$ for a suitable $j$, and is therefore a Noetherian $R$-module. It follows that $M / N$ is a Noetherian $R$-module, hence $K / N$, and so also $K$, is finitely generated.

Lemma 1.5. Let $I$ and $J$ be ideals of a ring $R$ such that $\sqrt{I}=\sqrt{J}$ (in particular, we may take $J=\sqrt{I}$ ). Then the following two conditions are equivalent: (i) $R / I$ is Noetherian and $I$ is finitely generated, and (ii) $R / J$ is Noetherian and $J$ is finitely generated.

Proof. Assume that $R / I$ is Noetherian and that $I$ is finitely generated. Thus $\sqrt{I}$ is finitely generated. For some $m \geq 1$ we have $(\sqrt{I})^{m} \subseteq J \subseteq \sqrt{I}$. By Lemma 1.4 with $I_{j}=\sqrt{I}$ for all $j$, the ideal $J$ is finitely generated. Since the ring $R / \sqrt{J}$ is Noetherian, and since $\sqrt{J}$ is finitely generated, we obtain that all prime ideals of the ring $R / J$ are finitely generated. By Cohen's Theorem $[8,(3.4)], R / J$ is Noetherian.

Corollary 1.6. Let $I$ be an ideal of a ring $R$. Assume that $R / I$ is Noetherian and that $I^{n}$ is finitely generated for some $n \geq 1$. Then $I$ is finitely generated iff the ring $R / I^{n}$ is Noetherian.

Lemma 1.7. Let $I$ be an ideal of a ring $R$. Assume that the ring $R / I$ is Noetherian and that some power of $I$ is finitely generated. Then $\sqrt{I}$ also satisfies these two conditions. Moreover, I is finitely generated iff $\sqrt{I}$ is finitely generated.

Proof. Assume that $I^{n}$ is finitely generated. Since the ring $R / I$ is Noetherian, we have $(\sqrt{I})^{k} \subseteq I$ for some $k \geq 1$. Thus $I^{k n} \subseteq(\sqrt{I})^{k n} \subseteq I^{n}$. By Lemma 1.4, the ideal $(\sqrt{I})^{k n}$ is finitely generated. By Lemma $1.5, I$ is finitely generated iff $\sqrt{I}$ is finitely generated.

Lemma 1.8 reduces Question 0.1 to the case where $M$ is the maximal ideal of a quasilocal ring; we omit its standard proof.

Lemma 1.8. Suppose $M$ is a maximal ideal of a ring $R$ that is the radical of a finitely generated ideal. Then the ideal $M$ of $R$ is finitely generated iff the ideal $M R_{M}$ of $R_{M}$ is finitely generated.

Theorem 1.9. Let $I$ be an ideal of the ring $R$ such that $R / I$ is Noetherian and some power of $I$ is finitely generated. If $c I \neq(0)$ for all nonzero elements $c \in I$ (this holds if $R$ is reduced), and if $\sqrt{I}=\sqrt{x R}$ for some $x \in R$, then $I$ is finitely generated.

Proof. We have $x^{m} \in I$ for some $m \geq 1$. Replacing $x$ by $x^{m}$ we may assume that $x \in I$. Choose $n$ minimal so that the ideal $I^{n}$ is finitely generated. Assume that 
$n>1$. We may choose $k$ so that $I^{k} \subseteq x I$. Thus $I^{k+n-2} \subseteq x I^{n-1} \subseteq I^{n}$, and Lemma 1.4 shows that $x I^{n-1}$ is finitely generated - say $x I^{n-1}=x J$, where $J$ is a finitely generated subideal of $I^{n-1}$. We have $\left(0:_{I} x\right) \subseteq\left(0:_{I} I^{k+n-2}\right)$, and since $\left(0:_{I} I\right)=(0)$, then $\left(0:_{I} I^{k+n-2}\right)=(0)$ as well. Thus $\left(0:_{I} x\right)=(0)$, so that $x$ is not a zero-divisor on $I$. Hence $I^{n-1}=J$ is finitely generated, contradicting the minimality of $n$. Thus $n=1$, and $I$ is finitely generated.

Theorem 1.10. Let $I$ be an ideal of the ring $R$. Assume that $R / I$ is Noetherian and that some power of $I$ is finitely generated. Then $I$ is finitely generated in each of the following cases:

(1) $\sqrt{I}=\sqrt{(J, x)}$, where $J$ is a finitely generated ideal of $R$ and $x$ is a regular element $\bmod J$.

(2) $\sqrt{I}=\sqrt{\left(x_{1}, \ldots, x_{k}\right)}$, where $x_{1}, \ldots, x_{k}$ is a regular sequence of elements in $R(k \geq 1)$.

(3) $R$ is a reduced ring, and $\sqrt{I}=\sqrt{x R}$ for some $x \in R$.

Proof. Item (1) follows from Theorem 1.9 (applied to the ring $R / J$ ), and (3) also follows from Theorem 1.9. Item (2) is a particular case of item (1).

Remark 1.11. Theorem 1.10 and other results of this type can be combined with Lemmas 1.7 and 1.5 to produce further results. In Theorem 1.10, for example, we may replace the assumptions on $I$ that $R / I$ is Noetherian and that some power of $I$ is finitely generated by the same assumptions on $\sqrt{I}$.

Lemma 1.12. Let $x$ be a regular element in a ring $R$ such that $R / \sqrt{x R}$ is Noetherian. If some power of $\sqrt{x R}$ is finitely generated, then the ring $R / x R$ is Noetherian, and thus, in particular, $\sqrt{x R}$ is finitely generated.

Proof. By Theorem 1.10 (2) the ideal $\sqrt{x R}$ is finitely generated. By Lemma 1.5 the ring $R / x R$ is Noetherian.

Remark 1.13. We recall from [9] that the following conditions are equivalent for a ring $R$ : (1) Spec $R$ is Noetherian, (2) Each radical ideal of $R$ is the radical of a finitely generated ideal, (3) Each prime ideal of $R$ is the radical of a finitely generated ideal, (4) $R$ satisfies the ascending chain condition on prime ideals and over each ideal there are only finitely many minimal primes.

Lemma 1.14. Let $R$ be a reduced ring such that for each minimal prime $P$ the ring $R / P$ is Noetherian. Assume also that $R$ has only finitely many minimal primes or that each minimal prime is the radical of a finitely generated ideal. Then $R$ is Noetherian.

Proof. Remark 1.13 shows that in either of the two cases, $R$ has only finitely many minimal primes: $P_{1}, \ldots, P_{n}$. Since $R / P_{i}$ is Noetherian for each $i$ we conclude that $R \cong R /\left(\bigcap_{i=1}^{n} P_{i}\right)$ is Noetherian $[8,(3.16)]$.

Lemma 1.15. Let $R$ be a ring such that for each minimal prime $P$ the ring $R / P$ is Noetherian and some power of $P$ is finitely generated. Then the ideal $N=\sqrt{(0)}$ is nilpotent.

Proof. By Remark 1.13, $R$ has only finitely many minimal primes $P_{1}, \ldots, P_{k}$. Assume that the ideals $P_{1}^{n}, \ldots, P_{k}^{n}$ are finitely generated. We have $N^{k} \subseteq P_{1} \ldots P_{k}$, and hence $N^{k n} \subseteq\left(P_{1} \ldots P_{k}\right)^{n} \subseteq N$. Since $\left(P_{1} \ldots P_{k}\right)^{n}$ is finitely generated, it is nilpotent, as is $N$. 
Lemma 1.16. Let $R$ be a ring and let $x$ be a regular element of $R$. Assume for each minimal prime $P$ over $x$ that the ring $R / P$ is Noetherian and that some power of $P$ is finitely generated. Then the ideal $x R$ contains a power of its radical, and the ring $R / x R$ is Noetherian; in particular, $\sqrt{x R}$ is finitely generated.

Proof. By Remark 1.13, $x R$ has only finitely many minimal primes $P_{1}, \ldots, P_{k}$, and by Lemma $1.15, x R$ contains a power of its radical. Assume that the ideals $P_{1}^{n}, \ldots, P_{k}^{n}$ are finitely generated. If $M=\left(P_{1} \ldots P_{k}\right)^{n}$, then $M$ is a finitely generated $R$-module such that $P_{1}^{m_{1}} \ldots P_{k}^{m_{k}} M$ is finitely generated for any nonnegative integers $m_{1}, \ldots, m_{k}$ (Lemmas 1.1 and 1.2). By Lemma 1.4, the ideal $(\sqrt{x R})^{k n}$ is finitely generated. Since $R / \sqrt{x R}$ is Noetherian by Lemma 1.14 , we obtain by Lemma 1.12 that the ring $R / x R$ is Noetherian as well.

Theorem 1.17. Let $R$ be a reduced ring. Assume that each prime ideal of $R$ has a power that is finitely generated. Then $R$ is Noetherian.

Proof. By Lemma 1.14 it is enough to prove that $R / P$ is Noetherian for each minimal prime $P$. Thus we may assume that $R$ is an integral domain. Assume that $R$ is not Noetherian. Since Spec $R$ is Noetherian, there is a prime ideal $Q$ that is maximal in the set of prime ideals of $R$ so that $R / Q$ is not Noetherian. Replacing $R$ by $R / Q$ we may also assume that $R / P$ is Noetherian for any nonzero prime ideal $P$. Let $P$ be a nonzero prime ideal of $R$ and let $x$ be a nonzero element in $P$. By Lemma $1.16, P / x R$ is finitely generated, and so is $P$. It follows that $R$ is Noetherian.

It is easy to give an example of a zero-dimensional quasilocal ring $(R, M)$ such that $M$ is not finitely generated but $M^{2}=(0)$. Thus Theorem 1.17 does not hold for a general ring $R$.

Proposition 1.18. Let $R$ be a ring. (i) If each prime ideal of $R$ has a power that is finitely generated, then $R / \sqrt{(0)}$ is Noetherian and the ideal $\sqrt{(0)}$ is nilpotent. (ii) If $R / \sqrt{(0)}$ is Noetherian, then the following conditions are equivalent: (1) $R$ is Noetherian. (2) Each minimal prime of $R$ is finitely generated; (3) $\sqrt{(0)}$ is finitely generated; (4) $\sqrt{(0)}$ is nilpotent and the $R$-module $\sqrt{(0)} /(\sqrt{(0)})^{2}$ is finitely generated.

Proof. (i): By Theorem 1.17, $R / \sqrt{(0)}$ is Noetherian, and by Lemma $1.15, \sqrt{(0)}$ is nilpotent.

(ii): If $R / \sqrt{(0)}$ is Noetherian, we show that $R$ is Noetherian under each of the conditions (2)-(4). Assuming (2), let $P$ be a prime ideal of $R$, and let $P_{0}$ be a minimal prime contained in $P$. Since $R / \sqrt{(0)}$ is Noetherian, the ring $R / P_{0}$ is also Noetherian. Thus $P / P_{0}$ is finitely generated, and so is $P$. By Cohen's Theorem $R$ is Noetherian. Assuming (3) we may use Cohen's Theorem again since any prime ideal contains $\sqrt{(0)}$. Assuming (4), let $N=\sqrt{(0)}$ so that $N^{n}=(0)$ for some $n \geq 1$. We have $N=I+N^{2}$ for some finitely generated ideal $I$ contained in $N$. Thus $N=I+\left(I+N^{2}\right)^{2}=I+N^{4}=\ldots$. Since $N$ is nilpotent, we see that $N=I$. By (3) the ring $R$ is Noetherian.

Corollary 1.19. Let $I$ be an ideal of a ring $R$ such that the ring $R / I$ is Noetherian and such that some power of I is finitely generated. Then I is finitely generated iff each minimal prime over I is finitely generated. 
Proof. Let $I^{n}$ be finitely generated. By Corollary 1.6, $I$ is finitely generated iff $R / I^{n}$ is Noetherian. Now apply Proposition 1.18(ii) [(1)

Theorem 1.20. Let $R$ be a reduced semiquasilocal ring. If each prime ideal of $R$ of positive height has a power that is finitely generated, then $R$ is Noetherian.

Proof. By Lemma 1.14 and Theorem 1.17, it is enough to show that $R$ has only finitely many minimal primes. Thus we may assume that $(R, M)$ is quasilocal. If $\operatorname{dim} R=0$, then $R$ is a field. Assume that $\operatorname{dim} R>0$. The ideal $M$ has a finitely generated power, so $M=\sqrt{\left(m_{1}, \ldots, m_{k}\right)}$, where each $m_{i}$ is nonzero. Since $R$ is reduced, each $\left(0: m_{i}\right)$ is a radical ideal, $\bigcap_{i=1}^{k}\left(0: m_{i}\right)=(0)$, and the image of $m_{i}$ in the ring $R /\left(0: m_{i}\right)$ is regular for each $i$. It suffices to show that the $\operatorname{ring} R /\left(0: m_{i}\right)$ has only finitely many minimal primes for each $i$. Hence we may assume that $R$ contains a regular element $x$. Let $P$ be a minimal prime of $R$. Since each minimal prime over $x$ is of positive height, by Lemma 1.16 the ring $R / x R$ is Noetherian. It follows that the ideal $P+x R$ is finitely generated. Thus $P \subseteq I+x R$, where $I \subseteq P$ is a finitely generated ideal; we conclude that $P=I+x P$. In the ring $\bar{R}=R / \sqrt{I}$ we have $\bar{P} \subseteq \bigcap_{n=1}^{\infty} \bar{x}^{n} \bar{R}$. For each prime $Q$ of $\bar{R}$ the ring $\bar{R} / Q$ is a Noetherian local domain by Theorem 1.17, so, $\bigcap_{n=1}^{\infty} \bar{x}^{n} \bar{R} \subseteq Q$. Hence $\bigcap_{n=1}^{\infty} \bar{x}^{n} \bar{R}=(0)$, which implies that $P=\sqrt{I}$. By Remark 1.13, $R$ has only finitely many minimal primes.

The following corollaries are immediate from Theorem 1.20.

Corollary 1.21. If $(R, M)$ is a quasilocal one-dimensional reduced ring such that some power of $M$ is finitely generated, then $R$ is Noetherian.

Corollary 1.22. If $(R, M)$ is a one-dimensional quasilocal integrally closed domain having the property that some power of Mis finitely generated, then $R$ is a rank-one discrete valuation domain (DVR).

Question 0.2 has an easy affirmative answer if the property "finitely generated" is replaced by "invertible" (without assuming that $R / I$ is Noetherian); for if some power of an ideal $I$ is invertible (in particular, a principal ideal generated by a regular element), then $I$ is invertible, hence finitely generated. For invertible ideals, see $[4, \S 7]$. It would be interesting to know if there exists a non-finitely generated ideal $I$ in an integral domain such that $I^{2}$ is 2-generated.

Lemma 1.23. Suppose $M$ is a maximal ideal of an integrally closed domain $R$. If $M^{n}$ is finitely generated for some $n \geq 1$ and $M$ is divisorial, then $M$ is invertible and hence finitely generated.

Proof. Since $R$ is integrally closed, $\left(M^{n}: M^{n}\right)=R$ by [4, Theorem 9.3 (c)]. Thus we have $(M: M) \subseteq\left(M^{n}: M^{n}\right)=R$. Since $M$ is divisorial, $(R: M) \neq R$. Thus $(R: M) M \nsubseteq M$, which implies that $(R: M) M=R$, so $M$ is invertible. (See [2, Theorem 1.3].)

Theorem 1.24. Let $R$ be a ring and let $M$ be a maximal ideal of $R$. Assume that some power of $M$ is finitely generated. Then $M$ is finitely generated in each of the following cases:

(1) $M$ is a minimal prime over a principal ideal, and $c M \neq(0)$ for each nonzero $c \in M$. 
(2) $M$ is a minimal prime over the ideal $I+x R$, where $I$ is a finitely generated ideal of $R$ and $x$ is a regular element $\bmod I$.

(3) $M$ is a minimal prime over the ideal generated by a nonempty regular sequence of elements in $R$.

(4) $R$ is a reduced ring and either $M$ is a minimal prime over a principal ideal, or ht $M \leq 1$.

(5) $R$ is an integral domain, $M$ is not divisorial, and either $M$ is a minimal prime over a 2-generated ideal, or ht $M \leq 2$.

(6) $R$ is an integrally closed domain, and either $M$ is a minimal prime over a 2 -generated ideal, or ht $M \leq 2$.

Proof. By Lemma 1.8, in parts (1)-(4) we may assume that $R$ is quasilocal. Part (1) follows from Theorem 1.9, and parts (2) and (3) from Theorem 1.10. For part (4), if $M$ is a minimal prime over a principal ideal, then $M$ is finitely generated by part (1). If ht $M=0$, then $R$ is a field. If ht $M=1$, then $R$ is Noetherian by Corollary 1.21. For part (5), first let $M$ be a minimal prime over $(x, y)$. Since $M$ is not divisorial, it follows that the ideal $M / x R$ of the ring $R / x R$ is not an annihilator. We conclude by (1) that $M$ is finitely generated. If ht $M \leq 2$, we may assume by (4) that $M$ is not a minimal prime over a principal ideal. Hence, if $0 \neq x \in M$, then $\operatorname{ht}(M / \sqrt{x R})=1$, and the ring $R_{M} / \sqrt{x R_{M}}$ is Noetherian by Corollary 1.21. Thus $M$ is a minimal prime over $(x, y)$ for some $y \in M$, which implies that $M$ is finitely generated. Part (6) follows from (5) and from Lemma 1.23.

Corollary 1.25. Let $R$ be a ring and let $M$ be a maximal ideal of $R$. If some power of $M$ is finitely generated and if, for some ideal $I$ of $R$ and $x \in R$, the ideal $M$ is a minimal prime over $I+x R$, then the $R$-module $M /(\sqrt{I})$ is finitely generated.

Proof. Replacing the ring $R$ by $R / \sqrt{I}$ we may assume that $R$ is reduced and that $M$ is a minimal prime over $x$. By Theorem 1.24 (1), $M$ is finitely generated.

\section{Semigroups AND MONOID RINGS}

All the semigroups and monoids in this section are commutative and are written in additive notation. If $S$ is a semigroup, we denote by $\tilde{S}$ the monoid $S \cup\{0\}$ obtained by adjoining a zero element to $S$. For a semigroup $S$ and a field $K$, let $K[X ; \tilde{S}]$ be the monoid ring of $\tilde{S}$ over $K$. If $S$ is cancellative and torsion-free, it is well known that $K[X ; \tilde{S}]$ is an integral domain [5, page 82]. For an additive semigroup $S$ and a positive integer $m$, we use $m S$ to denote the ideal consisting of all sums $s_{1}+s_{2}+\cdots+s_{m}$, where each $s_{i}$ is an element of $S$.

Proposition 2.1. Let $S$ be a semigroup with $0 \notin S$ and let $K$ be a field. Let $M$ be the ideal of the monoid ring $K[X ; \tilde{S}]$ generated by $\left\{X^{s}: s \in S\right\}$, and let $n$ be a positive integer. Then $M^{n}$ is finitely generated as an ideal of $K[X ; \tilde{S}]$ if and only if $n S$ is finitely generated as an ideal of the semigroup $S$. In particular, with $n=1$, the maximal ideal $M$ of $K[X ; \tilde{S}]$ is finitely generated if and only if $S$ is finitely generated as an ideal of $S$.

Proof. By definition $M^{n}$ is generated by $\left\{X^{s}: s \in n S\right\}$. Moreover, $M^{n}$ is finitely generated if and only if it is generated by $\left\{X^{a}: a \in A\right\}$ for some finite subset $A$ of $n S$. For any subset $A$ of $S$ and any $s \in S$ the element $X^{s}$ belongs to the ideal in $K[X ; \tilde{S}]$ generated by $\left\{X^{a}: a \in A\right\}$ iff $s$ belongs to the ideal of $S$ generated by $A$. The proposition follows. 
Proposition 2.2. Let $S$ be a semigroup and $n$ a positive integer. If $n S$ is finitely generated as a semigroup, then $n S$ is also finitely generated as an ideal of $S$. Conversely, if $\bigcap_{m=1}^{\infty} m S=\emptyset$ and if $n S$ is finitely generated as an ideal of $S$, then $n S$ is contained in a finitely generated subsemigroup of $S$; in particular, if $n=1$, then $S$ is a finitely generated semigroup.

Proof. If $A$ is a set of generators of $n S$ as a semigroup, then $A$ generates $n S$ as an ideal of $S$. Conversely, assume that $\bigcap_{m=1}^{\infty} m S=\emptyset$ and that $A=\left\{a_{1}, \ldots, a_{k}\right\}$ generates $n S$ as an ideal; thus $n S=\bigcup_{i=1}^{k}\left(a_{i}+\tilde{S}\right)$. For each $i$ let $a_{i}=\sum_{j=1}^{n} \alpha_{i j}$, where the elements $\alpha_{i j}$ are in $S$. Let $B$ be the set of all the elements $\alpha_{i j}$, and let $T$ be the subsemigroup of $S$ generated by $B$. We show that $n S \subseteq T$. Let $x \in n S$. Since $\bigcap_{i=1}^{\infty} i S=\emptyset$, we can choose $m \geq n$ maximal so that $x=\left(\sum_{i=1}^{m} b_{i}\right)+s$, where $b_{i} \in B$ and $s \in \tilde{S}$. If $s \neq 0$, then $\left(\sum_{i=1}^{n-1} b_{i}\right)+s=\left(\sum_{i=1}^{n} c_{i}\right)+t$, where $c_{i} \in B$ and $t \in \tilde{S}$. Thus $x=\left(\sum_{i=1}^{n} c_{i}\right)+\left(\sum_{i=n}^{m} b_{i}\right)+(s+t)$, contradicting the maximality of $m$. It follows that $s=0$ and that $x \in T$. Hence $n S \subseteq T$, as claimed.

As seen from the proof of Proposition 2.2, if $\bigcap_{m=1}^{\infty} m S=\emptyset$ and $A$ is a subset of $S$, then $A$ generates $S$ as a semigroup iff $A$ generates $S$ as an ideal of $S$.

Theorem 2.3. Let $S$ be a cancellative commutative semigroup such that $\bigcap_{m=1}^{\infty} m S$ $=\emptyset$ and such that for some integer $n \geq 1$ the ideal $n S$ of $S$ is finitely generated. Then $S$ is finitely generated as a semigroup.

Proof. By Proposition 2.2, the semigroup $n S$ is contained in a semigroup that is generated by a finite set $\left\{a_{1}, \ldots, a_{k}\right\}$. We show that $S \backslash 2 S$ is finite. Otherwise, let $x_{1}, x_{2}, \ldots$ be an infinite sequence of distinct elements in $S \backslash 2 S$. Let $c$ be any element in $n S$. For each $i$ we have in $S \cup\{0\}$ the equation $c+x_{i}=\sum_{j=1}^{k} n_{i j} a_{j}$, where each $n_{i j}$ is a nonnegative integer. Since the monoid $\left(\mathbb{N}^{k},+\right)$ is Noetherian, we see that there are integers $i<r$ such that $n_{i j} \leq n_{r j}$ for all $1 \leq j \leq k$. Hence $c+x_{r}=c+x_{i}+x$, where $x \in S$. Since $S$ is cancellative, we obtain $x_{r}=x_{i}+x$ contradicting the assumption that $x_{r} \notin 2 S$.

We now show that the semigroup $S$ is generated by $S \backslash 2 S$. Indeed, if $x \in S$, then there is an integer $m \geq 1$ such that $x \in m S \backslash(m+1) S$. If $x=s_{1}+\cdots+s_{m}$ in $S$, then $s_{1}, \ldots, s_{m}$ are in $S \backslash 2 S$.

As seen from the proof of Theorem 2.3, if $S$ is a cancellative semigroup such that $\bigcap_{m=1}^{\infty} m S=\emptyset$, then $S$ is generated as a semigroup by $S \backslash 2 S$; moreover, $S$ is finitely generated iff $S \backslash 2 S$ is finite since a subset of $S$ generates $S$ as a semigroup iff it contains $S \backslash 2 S$.

In the next proposition we apply Theorem 2.3 to Archimedean monoids. We recall that an ordered monoid $(S,<)$ is Archimedean if for any two elements $a>$ $0, b>0$ of $S$, there exists a positive integer $k$ such that $k a>b$.

Proposition 2.4. Let $(S,<)$ be an Archimedean monoid such that $S^{*}=S \backslash\{0\}$ consists of positive elements. Suppose for some integer $n \geq 1$ the ideal $n S^{*}$ of $S$ is finitely generated. Then $S$ is finitely generated as a monoid.

Proof. We first show that $S^{*}$ has a smallest element. Assume the ideal $n S^{*}$ of $S$ is generated by $\left\{a_{0}, a_{1}, \ldots, a_{k}\right\}$. We may assume that $a_{0}<a_{1}<\cdots<a_{k}$. Then $a_{0}$ is the smallest element of $n S^{*}$. Moreover, if we write $a_{0}=c_{1}+c_{2}+\cdots+c_{n}$, where each $c_{i} \in S^{*}$ and $c_{1} \leq c_{2} \leq \cdots \leq c_{n}$, then $a_{0} \geq n c_{1}$. Hence $a_{0}=n c_{1}$. If $c \in S^{*}$, then $n c \in n S^{*}$, so $n c \geq a_{0}=n c_{1}$ and $c \geq c_{1}$. Therefore $c_{1}$ is the smallest 
element of $S^{*}$. Since $m c_{1}$ is the smallest element of $m S^{*}$ for each $m$ and since the Archimedean property of $S$ implies that no element of $S$ is greater than each $m c_{1}$, we see that $\bigcap_{m=1}^{\infty} m S^{*}=\emptyset$. By Theorem 2.3 we conclude that $S$ is finitely generated.

As a result of Proposition 2.1 and Theorem 2.3 we obtain:

Theorem 2.5. Let $K$ be a field, and $S$ be a cancellative semigroup satisfying the property $\bigcap_{m=1}^{\infty} m S=\emptyset$. Let $M$ be the ideal of the monoid ring $K[X ; \tilde{S}]$ generated by $\left\{X^{s}: s \in S\right\}$. If some power of the maximal ideal $M$ is finitely generated, then $M$ is finitely generated.

Corollary 2.6. Let $K$ be a field and let $R$ be a $K$-subalgebra of the polynomial ring $K\left[X_{1}, \ldots, X_{n}\right]$ that is generated by a set $L$ of monomials. Let $M$ be the ideal of $R$ generated by $L$. If some power of $M$ is finitely generated, then $R$ is finitely generated as a $K$-algebra and $M$ is finitely generated as an ideal of $R$.

Proof. Let $S$ be the subsemigroup of $\left(\mathbb{N}^{n},+\right)$ generated by the elements $\left\{\left(i_{1}, \ldots, i_{n}\right)\right.$ : $\left.X_{1}^{i_{1}} \ldots X_{n}^{i_{n}} \in L\right\}$. Since $R$ is isomorphic as a $K$-algebra to the monoid algebra $K[X ; \tilde{S}]$, the corollary follows from Theorem 2.5.

\section{EXAMPLES AND FURTHER REMARKS}

As mentioned after Theorem 1.17, it is easy to give an example of a commutative ring $R$ having a non-finitely generated maximal ideal $M$ such that $M^{2}=(0)$, so, in particular, $M^{2}$ is finitely generated. There are also examples where an integral domain $R$ has a non-finitely generated prime ideal $P$ such that $P^{2}$ is finitely generated. A general method for constructing such examples is as follows:

Example 3.1. Let $T$ be a non-Noetherian integral domain and let $I$ be a nonfinitely generated ideal of $T$. Let $R$ denote the subring $R=T\left[X^{3}, X^{4}, I X^{5}\right]$ of the polynomial ring $T[X]$. Then $P=\left(X^{3}, X^{4}, I X^{5}\right)$ is a non-finitely generated prime ideal of $R$ with the property that $P^{2}=\left(X^{6}, X^{7}, X^{8}\right)$ is finitely generated. Note that in this construction we have $R / P \cong T$, so $P$ is a nonmaximal prime ideal.

Example 3.2. For each $d$ with $3 \leq d \leq \infty$ there exists a $d$-dimensional integral domain $T$ having a nonfinitely generated maximal ideal $P$ with ht $P=d$ such that $P^{2}$ is 3 -generated and such that there exists a 2-generated ideal $I$ contained in $P$ with $I^{2}=P^{2}$. If $d=\infty$, then $T$ can be chosen to be a monoid domain $K[X ; S]$ over a field $K$, where $P$ is the ideal generated by $\left\{X^{s}: s \in S\right\}$.

Let $K$ be a field and let $\left\{X_{n}\right\}_{n \geq 1}$ be independent indeterminates over $K$. First assume that $d$ is finite and let $L$ be the field $K\left(X_{n}^{2} / X_{3}^{2}\right)_{n \geq d+1}$. Set

$$
T=L\left[X_{n}, \frac{X_{1} X_{i}}{X_{2}^{m}}, \frac{X_{2} X_{i}}{X_{1}^{m}}\right]_{n \geq 1, m \geq 1, i \geq 3} .
$$

Let $P=\left(X_{n}\right)_{n \geq 1}$. It is easy to show that $P$ is a maximal ideal of $T$ and that $P^{2}=\left(X_{1}, X_{2}\right)^{2}$. Assume that $P$ is finitely generated. Then $X_{n+1} \in\left(X_{1}, \ldots, X_{n}\right)$ in $T$ for some $n \geq 2$. By considering any nonzero element of $T$ as a rational function in the indeterminates $\left\{X_{n}\right\}_{n \geq 3}$ over the field $K\left(X_{1}, X_{2}\right)$ and by using its total degree in $\left\{X_{n}\right\}_{n \geq 3}$, we view $T$ as an $\mathbb{N}$-graded ring. A nonzero element 
of $L$ is of degree 0 . Since $X_{n+1}$ is homogeneous of degree 1 and since each element of $T$ is a linear combination over $L$ of finite products of elements in the set $\left\{X_{n}, \frac{X_{1} X_{i}}{X_{2}^{m}}, \frac{X_{2} X_{i}}{X_{1}^{m}}\right\}_{n \geq 1, m \geq 1, i \geq 3}$, we obtain

$$
X_{n+1}=f_{0}+\left(f_{1} \frac{X_{1}}{X_{2}^{m}}+f_{2} \frac{X_{2}}{X_{1}^{l}}\right) X_{n+1},
$$

where $f_{0}$ is a linear combination of $\left\{X_{i}\right\}_{i \geq 3, i \neq n+1}$ with coefficients in the field $L\left(X_{1}, X_{2}\right), f_{1}, f_{2} \in L\left[X_{1}, X_{2}\right]$ and $m, l \geq 0$. By considering the $\mathbb{Z} / 2 \mathbb{Z}$-grading $T=T_{0} \oplus T_{1}$, where $T_{0}=T \cap L\left(X_{i}\right)_{i \neq n+1}\left[X_{n+1}^{2}\right]$ and $T_{1}=X_{n+1} T_{0}$, we obtain that

$$
f_{1} \frac{X_{1}}{X_{2}^{m}}+f_{2} \frac{X_{2}}{X_{1}^{l}}=1
$$

Multiply this equation by $X_{1}^{l}$ to obtain that in $L\left[X_{1}, X_{2}\right]$ the polynomial $f_{1}$ is divisible by $X_{2}^{m}$; similarly, $f_{2}$ is divisible by $X_{1}^{l}$. Thus $1 \in\left(X_{1}, X_{2}\right)$ in $L\left[X_{1}, X_{2}\right]$, a contradiction. We conclude that $P$ is not finitely generated.

Let $\tilde{L}=K\left(X_{n} / X_{3}\right)_{n \geq d+1}$, let $D_{i}=\tilde{L}\left[X_{1}, X_{2}, \frac{X_{3}}{X_{1}^{i} X_{2}^{i}}, \ldots, \frac{X_{d}}{X_{1}^{i} X_{2}^{i}}\right]$, let $\tilde{T}$ be the union $\bigcup_{i=1}^{\infty} D_{i}$, and let $\tilde{P}$ be the ideal $\left(X_{1}, X_{2}, \frac{X_{3}}{X_{1}^{i} X_{2}^{i}}, \ldots, \frac{X_{d}}{X_{1}^{i} X_{2}^{i}}\right)_{i \geq 1}$ of $\tilde{T}$. Then $\tilde{T}$ is integral over $T$ and $\tilde{P}=\left(X_{1}, X_{2}\right) \tilde{T}=P \tilde{T}$, so $\tilde{T}_{\tilde{P}}$ is integral over $T_{P}$. Since each $D_{i}$ is a polynomial ring in $d$ indeterminates over the field $\tilde{L}$, we see that $\tilde{T}$ is the integral closure of $T$ and $\tilde{T}_{\tilde{P}}$ is the integral closure of $T_{P}$. To show $\operatorname{dim} T_{P}=d$, it suffices to show $\operatorname{dim} \tilde{T}_{\tilde{P}}=d$. Since $\tilde{T}$ is an ascending union of $d$-dimensional rings, $\operatorname{dim} \tilde{T}_{\tilde{P}} \leq d$. The ideal $Q=\left(\frac{X_{3}}{X_{1}^{i} X_{2}^{i}}, \ldots, \frac{X_{d}}{X_{1}^{i} X_{2}^{i}}\right)_{i \geq 1}$ of $\tilde{T}$ is of height $d-2$ and $\tilde{T} / Q$ is a polynomial ring in two variables over $\tilde{L}$. Hence $\operatorname{dim} \tilde{T}_{\tilde{P}}=d$.

If $d=\infty$, let $T$ be the monoid domain

$$
K[X ; S]=K\left[X_{n}, \frac{X_{1} X_{i}}{X_{2}^{m}}, \frac{X_{2} X_{i}}{X_{1}^{m}}\right]_{n \geq 1, m \geq 1, i \geq 3} .
$$

Remark 3.3. We obtain an example of a quasilocal domain $(R, M)$ having similar properties to those in Example 3.2 if we set $R=T_{P}$ and $M=P T_{P}$, where $T$ and $P$ are as defined in Example 3.2.

\section{REFERENCES}

[1] P. Eakin and A. Sathaye, Prestable ideals, J. Algebra 41 (1976), 439-454. MR 54:7449

[2] S. Gabelli, Complete integrally closed domains and t-ideals, Bolletino U.M.I (7) (1989), 327342. MR 90h:13019

[3] R. Gilmer, On factorization into prime ideals, Commentarii Math. Helvetici 47 (1972), 70-74. MR 46:5310

[4] R. Gilmer, Multiplicative Ideal Theory, Queen's Papers Pure Appl. Math. Vol 90, Kingston, 1992. MR 37:5198

[5] R. Gilmer, Commutative Semigroup Rings, Chicago Lectures in Math., Chicago, 1984. MR 85e:20058

[6] W. Heinzer, D. Lantz, and K. Shah, The Ratliff-Rush ideals in a Noetherian ring, Comm. in Algebra 20 (1992), 591-622. MR 93c: 13002

[7] I. Kaplansky, Commutative Rings, Revised Edition, The University of Chicago Press, 1974. MR 49:10674

[8] M. Nagata Local Rings, Interscience, New York, 1962. MR 27:5790 
[9] J. Ohm and R. Pendleton, Rings with Noetherian spectrum, Duke Math. J. 35 (1968), 631-639; Addendum, 875. MR 37:5201

[10] L. J. Ratliff, Jr., and D. E. Rush, Two notes on reductions of ideals, Indiana Univ. Math. J. 27 (1978), 929-934. MR 58:22034

Department of Mathematics, Florida State University Tallahassee, Florida 32306 4510

E-mail address: gilmer@math.fsu.edu

Department of Mathematics, Purdue University, West Lafayette, Indiana 47907-1395

E-mail address: heinzer@math.purdue.edu

Department of Mathematics, University of Haifa, Mount Carmel, Haifa 31905, Israel

E-mail address: mroitman@mathcs2.haifa.ac.il 